\title{
Harmine induces cell cycle arrest and mitochondrial pathway-mediated cellular apoptosis in SW620 cells via inhibition of the Akt and ERK signaling pathways
}

\author{
JIMING LIU ${ }^{1}$, QIANG LI ${ }^{1}$, ZHILONG LIU ${ }^{1}$, LIUMING LIN ${ }^{1}$, XIANGQIANG ZHANG ${ }^{2}$, \\ MINGRONG CAO $^{1}$ and JIANWEI JIANG ${ }^{2}$
}

\author{
${ }^{1}$ Department of General Surgery, First Affiliated Hospital, Jinan University, Guangzhou, Guangdong 510630; \\ ${ }^{2}$ Department of Biochemistry, Medical College, Jinan University, Guangzhou, Guangdong 510630, P.R. China
}

Received November 8, 2015; Accepted January 17, 2016

DOI: 10.3892/or.2016.4695

\begin{abstract}
Harmine, a $\beta$-carboline alkaloid isolated from the seeds of Peganum harmala, possesses both antitumor and anti-nociceptive effects and inhibits human DNA topoisomerase. However, no detailed data are available concerning the mechanisms of harmine in human colorectal carcinoma SW620 cells. In the present study, we demonstrated that harmine inhibited the proliferation of SW620 cells in a dose-dependent manner using MTT and clone formation assays, and the $\mathrm{IC}_{50}$ value of harmine on the growth inhibition of SW620 cells for $48 \mathrm{~h}$ was $5.13 \mu \mathrm{g} / \mathrm{ml}$. PI staining showed that harmine altered the cell cycle distribution by decreasing the proportion of cells in the G0-G1 phase and increasing the proportion in the S and G2-M phase. The expression level of cyclin D1 was decreased, while the expression of cyclin A, E2 and B1, CDK1/cdc2, Myt-1 and p-cdc2 (Tyr15) were increased, which was in accordance with the $\mathrm{S}$ and $\mathrm{G} 2 / \mathrm{M}$ phase arrest. Hoechst 33258 staining revealed nuclear fragmentation, chromosomal condensation and cell shrinkage in the SW620 cells treated with harmine. Flow cytometry revealed that the percentage of apoptotic sub-G1 cells increased from 7.19 to $26.58 \%$, while in the control group, sub-G1 cells only increased from 1.53 to $1.60 \%$. Furthermore, early and late apoptotic cells were increased from 11.96 to $26.38 \%$ when incubated with the indicated concentration of harmine for $48 \mathrm{~h}$, while in the control group, $<8 \%$ of cells underwent apoptosis. JC-1 staining revealed that harmine decreased mitochondrial transmembrane potential $(\Delta \Psi \mathrm{m})$. The apoptosis of SW620 cells was also detected by western blot analysis, showing caspase- 3 and -9 , and PARP activation; the
\end{abstract}

Correspondence to: Dr Mingrong Cao, Department of General Surgery, First Affiliated Hospital, Jinan University, Guangzhou, Guangdong 510630, P.R. China

E-mail: tcaomr@jnu.edu.cn

Dr Jianwei Jiang, Department of Biochemistry, Medical College, Jinan University, Guangzhou, Guangdong 510630, P.R. China E-mail:jjw703@163.com

Key words: colon carcinoma, harmine, Akt, cell cycle, apoptosis downregulation of Bcl-2, Mcl-1, Bcl-xL; and the upregulation of Bax. The expression of p-ERK, p-Akt (Ser473) and p-Akt (Thr308) was inhibited, and phosphorylation of downstream targets of Akt, such as p-FoxO3a and p-GSK-3 $\beta$ were also attenuated. In conclusion, harmine induces cell cycle arrest and mitochondrial pathway-mediated cellular apoptosis in SW620 cells via inhibition of the Akt and ERK signaling pathways.

\section{Introduction}

Colon carcinoma is one of the most common and aggressive malignant tumors worldwide (1). Currently, treatments for colon carcinoma are mainly surgery and chemotherapy, but the curative effect of existing chemotherapeutic drugs is not good, and they have numerous side-effects, including myelosuppression, neutropenia and thrombocytopenia (2). Therefore, the exploration of a new approach, such as novel drugs with specific effects on colon carcinoma treatment is urgently needed.

Harmine, a $\beta$-carboline alkaloid isolated from the seeds of Peganum harmala (Fig. 1), has been traditionally used for ritual and medicinal preparations in the Middle East, Central Asia and South America (3). Previous research has shown that harmine plays roles in anticancer treatments (4-6) and possesses anti-leishmanial properties (7) and antiviral effects (8) via inhibition of DYRK1A substrate phosphorylation. This compound was also found to interfere with neuritogenesis in cultured hippocampal neurons (9) and inhibit angiogenesis (10), telomerase activity (5) and mitochondrial signaling pathways $(6,11)$, in addition to inducing DNA single- or double-strand breaks (12). It has been reported that harmine activates both the intrinsic and extrinsic pathways of apoptosis and regulates various transcription factors and pro-inflammatory cytokines in B16F-10 cells (6). Furthermore, the in vivo anti-angiogenic activity of harmine was studied using B16F-10 melanoma cells in C57BL/6 mice. The results showed that harmine decreased tumor capillary formation and inhibited angiogenesis (4). Harmine was also shown to induce apoptosis and suppress tumor cell proliferation through the downregulation of cyclooxygenase-2 expression in gastric cancer (13). Additionally, harmine can upregulate p21 and p27, enhance the formation 
of complexes with the G1-S phase CDKs and cyclins, and induce G1 arrest to stall cancer progression in human breast cancer MCF7 (p53 wild-type), MCF7 (p53 knockdown) and MDA-MB-468 (p53 mutant) cells (14). In cytotoxicity assays, harmine exhibited a strong inhibitory effect on the growth and proliferation of carcinoma cells, whereas it had no significant effects on quiescent fibroblasts (15). However, no detailed data are available in regards to the growth inhibition of human colon carcinoma cells. In the present study, we investigated the effect of harmine on the growth of human SW620 cells.

\section{Materials and methods}

Materials. Harmine was purchased from the Xi'an Feida Bio-Tech Co., Ltd. (Xi'an, China). Fetal bovine serum (FBS), RPMI-1640 medium, trypsin and EDTA were purchased from Gibco-BRL (Gaithersburg, MD, USA); Cell Counting Kit-8 (CCK-8/WST-8 kit) was purchased from Dojindo, Japan. Dimethyl sulfoxide (DMSO), Annexin V-fluorescent isothiocyanate (FITC) and propidium iodide (PI) were purchased from Sigma-Aldrich (St. Louis, MO, USA). Antibodies against Bax, Bcl-2, Bcl-xL, Mcl-1, caspase-9 and -3, poly(ADP-ribose) polymerase (PARP), cleaved PARP, cyclin A, B1, D1 and E2, CDK4, cdc2, p-cdc2 (Tyr15), Myt-1, Akt, p-Akt (Ser473), p-Akt (Thr308), GSK3 $\alpha / \beta$, p-GSK3 $\beta$ (Ser9), FoxO3a, p-FoxO3a (Ser318/321) and GAPDH as well as all secondary antibodies were purchased from Cell Signaling Technology Ltd. (Danvers, MA, USA).

Cell culture and treatment. Cells were cultured in RPMI-1640 medium supplemented with $10 \% \mathrm{FBS}$ at $37^{\circ} \mathrm{C}$ in a humidified atmosphere containing $5 \% \mathrm{CO}_{2}$. Harmine was dissolved in DMSO and diluted to appropriate concentrations with culture medium. The final concentration of DMSO in the culture medium did not exceed $0.1 \%$.

CCK-8 assay. CCK-8 testing was used to monitor cell proliferation. Cells were plated at a density of 5,000 cells/well in 96-well plates. After $24 \mathrm{~h}$ in culture, cells were treated with harmine at the final concentrations of $0,0.625,1.25,2.50,5.00$, 10.00 and $20.00 \mu \mathrm{g} / \mathrm{ml}$ for $48 \mathrm{~h}$. Control cells were treated with DMSO. After cells were incubated with harmine for $48 \mathrm{~h}, 10 \mu \mathrm{l}$ CCK-8 was added to each well. Absorbance was detected with an enzyme calibrator at $570 \mathrm{~nm}$ and then optical density (OD) values were measured. Inhibition of cell growth was computed as the percentage of viable cells compared with the control: Percentage $(\%)=\left(\mathrm{OD}_{\text {control }}-\mathrm{OD}_{\text {treatment }}\right) / \mathrm{OD}_{\text {control }} \times 100 \%$. Experiments were performed in triplicate.

Clone formation assay. A clone formation assay was used to evaluate the effects of harmine on the proliferation of SW620 cells. Cells were first cultured in 12-well microplates (300 cells/well) in $2.0 \mathrm{ml}$ of complete RPMI-1640 for $24 \mathrm{~h}$. Then, the cells were treated with the indicated concentrations of harmine for 7 days. Finally, the cells were stained with crystal violet for $20 \mathrm{~min}$. A digital camera captured images of the colonies as previously described (16).

Fluorescence microscopy assay. Harmine-induced apoptosis in SW620 cells was assessed by Hoechst 33258 staining.

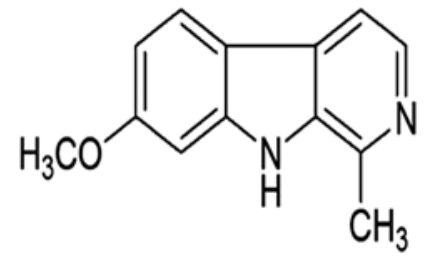

Figure 1. Chemical structure of harmine.

Following treatment with the indicated concentrations of harmine for $48 \mathrm{~h}$, the cells were harvested and smeared on slides. The slides were air-dried, fixed in methanol-acetone $(3: 1, \mathrm{v} / \mathrm{v})$ and stained with Hoechst $33258(5 \mu \mathrm{g} / \mathrm{ml})$ at $37^{\circ} \mathrm{C}$ in the dark for $20 \mathrm{~min}$. Nuclear morphology was examined using fluorescence microscopy (DFC480; Leica Microsystems, Wetzlar, Germany) to identify cells undergoing apoptosis.

Flow cytometry. SW620 cells at a density of 20,000 cells/well were incubated in 6-well plates for $48 \mathrm{~h}$ with the indicated concentrations of harmine. After incubation, the cells were harvested, washed with phosphate-buffered solution (PBS), and fixed in $70 \%$ ice-cold ethanol overnight. Then, the fixed cells were incubated with $20 \mathrm{U} / \mathrm{ml}$ RNase I and $50 \mu \mathrm{g} / \mathrm{ml}$ PI for $30 \mathrm{~min}$. The DNA content was determined by flow cytometry (FCM; Beckman Coulter, Fullerton, CA, USA). Apoptotic cells were identified by the sub-G1 phase in the cell cycle distribution. For assessment of the apoptotic rate, Annexin V-FITC/ PI staining was performed according to the manufacturer's protocol. The cells that were Annexin V-positive and PI-negative were defined as early apoptotic cells, and cells that were both Annexin V and PI-positive were defined as late apoptotic cells. The apoptotic rate was measured by FCM (Becton-Dickinson, USA) using CellQuest software.

Membrane potential of the mitochondria $(\Delta \Psi \mathrm{m})$. Changes in the membrane potential of mitochondria were analyzed with JC-1 staining. SW620 cells were treated with $2.50 \mu \mathrm{g} / \mathrm{ml}$ harmine for $48 \mathrm{~h}$, and then the cells were harvested, washed with PBS, fixed in JC-1 at $37^{\circ} \mathrm{C}$ in the dark for $30 \mathrm{~min}$, then harvested and smeared on slides. Changes in $\Delta \Psi \mathrm{m}$ were measured with a fluorescence microscope according to the manufacturer's protocol.

Western blot analysis. Total protein was extracted by incubation of cell pellets with lysis buffer. The protein concentration was determined using the BCA assay (Sigma) according to the manufacturer's instructions. Cell lysates were electrophoresed on $10-15 \%$ sodium dodecyl sulfate-polyacrylamide gel electrophoresis (SDS-PAGE) gels. The protein bands were then transferred to polyvinylidene difluoride (PVDF) membranes. After blocking with 5\% dried skimmed milk, the membranes were incubated overnight at $4^{\circ} \mathrm{C}$ with the appropriate primary antibody. Then, the membranes were washed three times in Tris-buffered saline and Tween-20 (TBST) and incubated for $1 \mathrm{~h}$ at room temperature with a secondary antibody conjugated to horseradish peroxidase. After washing in TBST, the bound antibody complex was detected using an ECL chemiluminescence reagent and X-ray film (Kodak, Rochester, NY, USA). 


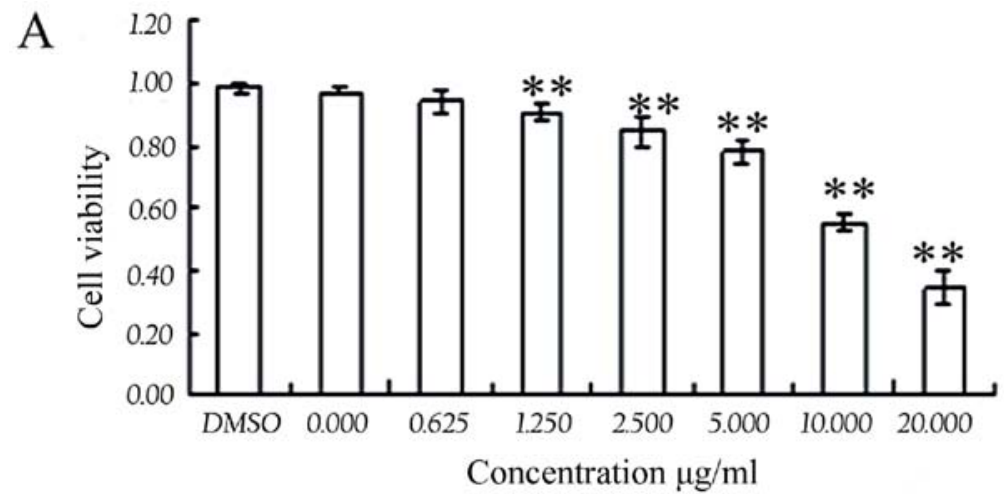

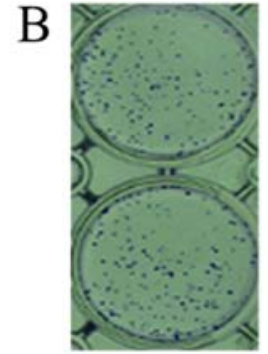

DMSO

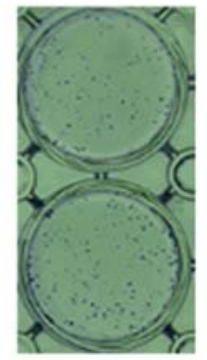

$0 \mu \mathrm{g} / \mathrm{ml}$

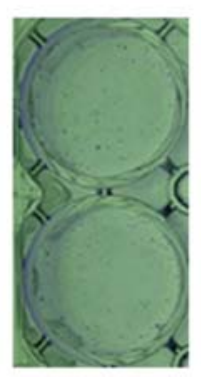

$0.625 \mu \mathrm{g} / \mathrm{ml}$

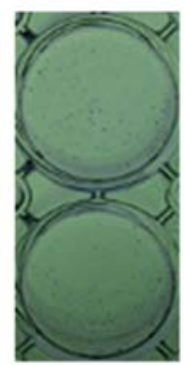

$1.25 \mu \mathrm{g} / \mathrm{ml}$

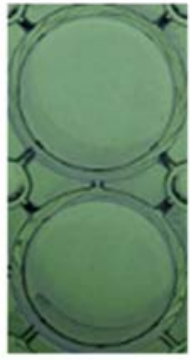

$2.50 \mu \mathrm{g} / \mathrm{ml}$

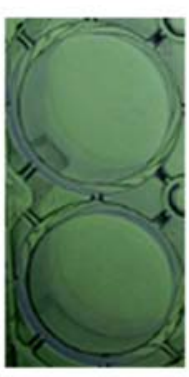

$5.00 \mu \mathrm{g} / \mathrm{ml}$

Figure 2. Harmine dose-dependently decreases the cell viability in SW620 cells. (A) Effects of harmine on the cell viability of SW620 cells. The indicated concentrations of harmine were added and the cells were incubated for $48 \mathrm{~h}$. Viability was measured by a CCK-8 assay. ${ }^{* *} \mathrm{P}<0.05$ vs. the control group. (B) Clone formation assay was used to evaluate the effects of harmine on the proliferation of SW620 cells. SW620 cells were treated with $0,0.625,1.25,2.50$ and $5.00 \mu \mathrm{g} / \mathrm{ml} \mathrm{harmine}$ for 7 days and stained with crystal violet for $20 \mathrm{~min}$. Results represented three independent experiments.

Statistical analysis. Data were analyzed by ANOVA and Student's t-tests. These analyses were performed using SPSS 13.0 software. All results are expressed as the means \pm standard error (SE) of three independent experiments. Differences with $\mathrm{P}<0.05$ were considered to be statistically significant.

\section{Results}

Harmine inhibits SW620 cell proliferation. To study the effects of harmine on cell proliferation, we used a CCK-8/ WST-8 assay to analyze the proliferation of the SW620 cells. After cells were incubated with the indicated concentrations of harmine for $48 \mathrm{~h}$, cell viability was markedly decreased (Fig. 2A). Harmine inhibited the growth of SW620 cells in a dose-dependent manner ( $\mathrm{P}<0.05$ vs. control), with a half-maximal inhibitory concentration $\left(\mathrm{IC}_{50}\right)$ value of $5.13 \mu \mathrm{g} /$ $\mathrm{ml}$. We further assessed the effect of harmine on the proliferation of SW620 cells using a clone formation assay. The results showed that harmine strongly inhibited SW620 cell proliferation in a dose-dependent manner after treatment with the indicated concentrations of harmine for 7 days (Fig. 2B).

Harmine alters the cell cycle and modulates the expression of cell cycle regulatory proteins in SW620 cells. Flow cytometry with a PI staining assay was used to analyze the effects of harmine on the cell cycle distribution. As shown in Fig. 3A, a significant population of cells was arrested in the $\mathrm{S}$ phase (38.3-50.1\%), compared to $38.3 \%$ in the DMSO-treated controls and the G2/M phase (9.7-20.0\%), compared to $5.5 \%$ in the DMSO-treated controls after treatment with harmine at the indicated concentrations for $48 \mathrm{~h}$. The population of cells in the S and G2-M phase increased, and the population of cells in the G1 phase decreased with increasing concentrations of harmine. These results indicate that cell cycle distribution was significantly arrested in the $\mathrm{S}$ and $\mathrm{G} 2 / \mathrm{M}$ phases upon harmine treatment.

As significant cell cycle arrest was observed with harmine treatment, we next assessed the effect of harmine on cell cycle regulatory proteins by western blot analysis. As shown in Fig. 3B, the expression of the $\mathrm{G} 1$ phase-related protein as cyclin D1, was significantly reduced after treatment with harmine for $48 \mathrm{~h}$. However, the expression levels of S- and G2/M phase-related proteins, such as cyclin A, B1 and E2, increased, and those of $\mathrm{G} 2 / \mathrm{M}$ phase-related proteins, such as cdc2, Myt-1 and p-cdc2 (Tyr15), were also increased.

Harmine induces apoptosis in SW620 cells. Apoptotic nuclear morphology was observed after Hoechst 33258 staining using fluorescence microscopy. After treatment with $5.00 \mu \mathrm{g} / \mathrm{ml}$ harmine for $48 \mathrm{~h}$, the SW620 cells began to exhibit apoptotic characteristics, such as cell shrinkage, nuclear condensation and fragmentation. In the control group, the cells were regular in morphology, grew fully in patches and were confluent, rarely sloughing off (Fig. 4A).

Apoptosis was also detected through a fluorescein Annexin V-FITC/PI double staining assay. The staining of cells with Annexin V-FITC and PI was used to distinguish and quantitatively determine the percentage of apoptotic cells. The SW620 cells underwent apoptosis after exposure to harmine at $1.25,2.50$ and $5.00 \mu \mathrm{g} / \mathrm{ml}$ for $48 \mathrm{~h}$, and the percentage of apoptotic cells stained by Annexin V-FITC is shown in Fig. 4B. The 


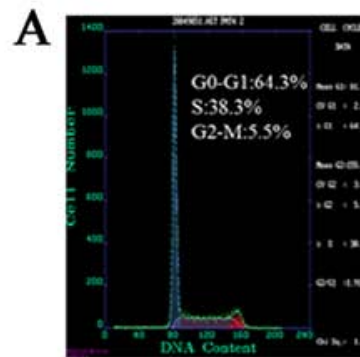

DMSO

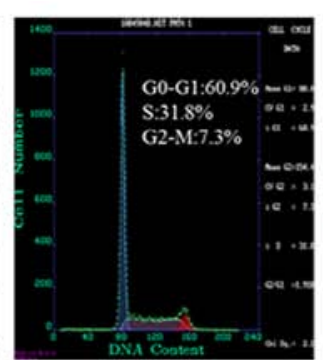

$0 \mu \mathrm{g} / \mathrm{ml}$

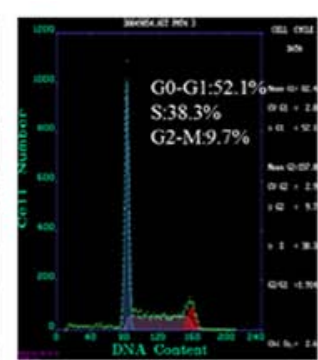

$1.25 \mu \mathrm{g} / \mathrm{ml}$

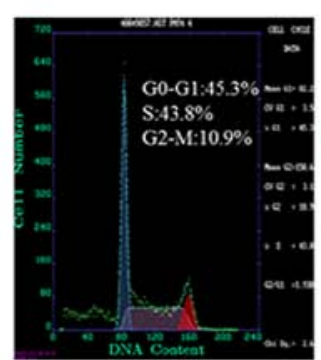

$2.50 \mu \mathrm{g} / \mathrm{ml}$

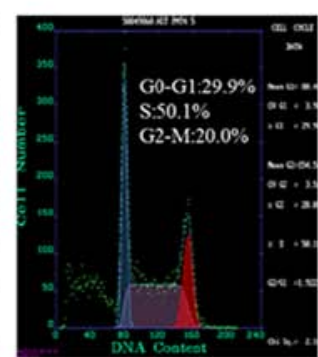

$5.00 \mu \mathrm{g} / \mathrm{ml}$

B

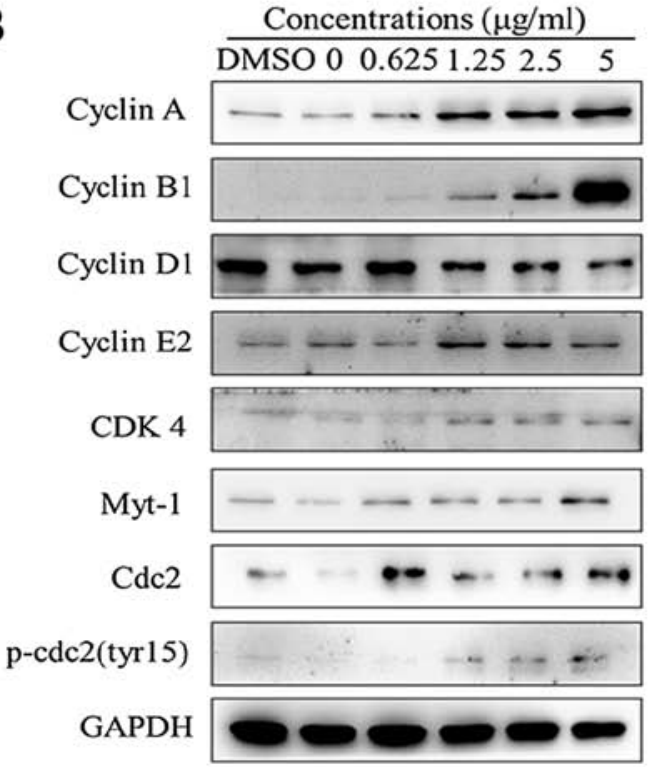

Figure 3. Harmine initiates cell cycle arrest in the S and G2/M phase in the SW620 cells. (A) PI staining demonstrated characteristic features of apoptosis in the harmine-exposed cells. The cell cycle distributions were detected by flow cytometry. (B) The expression of cell cycle-related proteins was analyzed by western blot analysis. The data represent one of three experiments that yielded similar results.

early and late apoptotic cells increased from 11.96 to $26.38 \%$ when incubated with the indicated concentrations of harmine for $48 \mathrm{~h}$, while in the control group, $<8 \%$ of cells were apoptotic.

PI staining and flow cytometric assays were used to investigate the percentage of sub-G1 cells, which indicates late apoptotic cells. The SW620 cells underwent apoptosis after being exposed to harmine at 1.25, 2.50 and $5.00 \mu \mathrm{g} / \mathrm{ml}$ for $48 \mathrm{~h}$, and the percentage of apoptotic cells as sub-G1 cells increased from 7.19 to $26.58 \%$, while in the control group, sub-G1 cells increased from 1.53 to $1.60 \%$ (Fig. 4C). The percentage of the sub-G1 fractions increased in a dose-dependent manner.

The caspases, a family of cysteine acid proteases, are known to act as important mediators of apoptosis and can cleave various cellular substrates. To study the mechanism by which harmine induces apoptosis, western blot analysis was conducted. Exposure of the SW620 cells to harmine resulted in cleavage of caspase- 3 and -9 , and PARP (Fig. 4D). Increased harmine concentrations resulted in the disappearance of the intact proteins and the appearance of proteolytic cleavage bands in a concentration-dependent manner, which indicates that the cells were undergoing apoptosis.

Effect of harmine on mitochondrial membrane potential $(\Delta \Psi m)$ and protein expression levels of the Bcl-2 family. Loss of $\Delta \Psi \mathrm{m}$ is a crucial step in the apoptotic process and is lethal to cells since it leads to the release of diverse pro-apoptotic factors, such as Smac and cytochrome $c$, from the mitochondria into the cytoplasm $(17,18)$. In the present study, we used the cationic dye JC-1 to determine the status of the mitochondria in SW620 cells. In non-apoptotic cells, JC-1 enters the negatively charged mitochondria, where it aggregates and turns red. However, in cells undergoing apoptosis where the $\Delta \Psi \mathrm{m}$ has collapsed, JC-1 exists as monomers in the cytosol and turns green. Our results showed that harmine induced a depletion of $\Delta \Psi \mathrm{m}$ in the SW620 cells (Fig. 5A).

Bcl-2 family proteins are key regulators of mitochondrial permeability (19). Therefore, we investigated whether the mitochondrial-mediated apoptosis in SW620 cells induced by harmine occurred as a result of the modulation of Bcl-2 family members. Harmine suppressed the expression of anti-apoptotic Bcl-2, Mcl-1 and Bcl-xL and increased the expression of pro-apoptotic Bax (Fig. 5B). As a result of these changes, the ratios of $\mathrm{Bcl}-2 / \mathrm{Bax}, \mathrm{Mcl}-1 / \mathrm{Bax}$ and $\mathrm{Bcl}-\mathrm{xL} / \mathrm{Bax}$ were significantly reduced during apoptosis.

Effects of harmine on the phosphorylation of Akt and its downstream targets in SW620 cells. To explore the mechanism of cellular apoptosis induced by harmine in SW620 cells, we examined the Akt signaling pathway. The results showed that increased harmine concentrations downregulated phosphorylation of Ser473-Akt and Thr308-Akt in a dose-dependent manner without affecting the total amount of Akt (Fig. 6). 


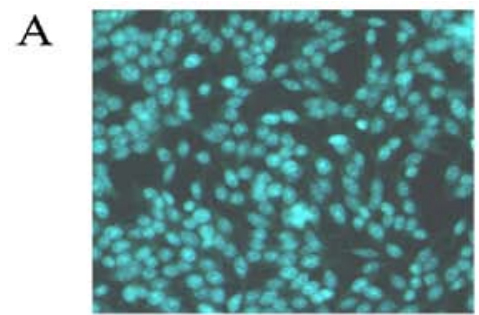

DMSO

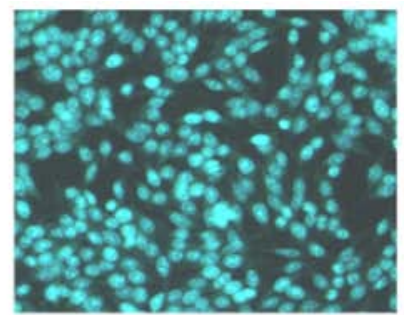

$0 \mu \mathrm{g} / \mathrm{ml}$

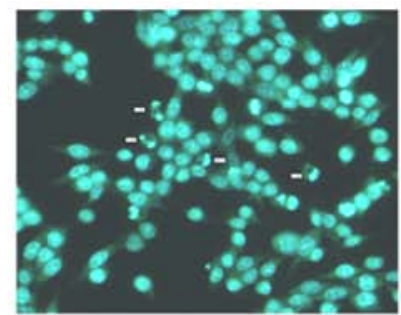

$5 \mu \mathrm{g} / \mathrm{ml}$
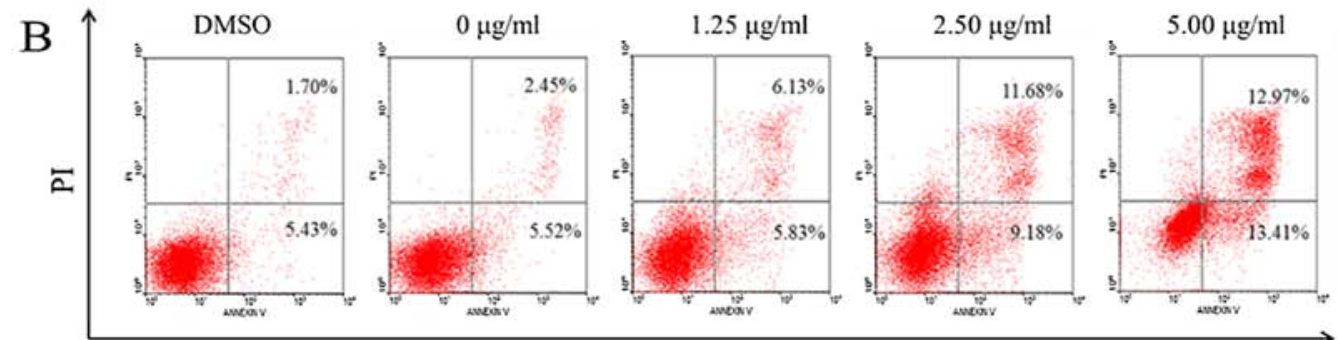

Annexin V
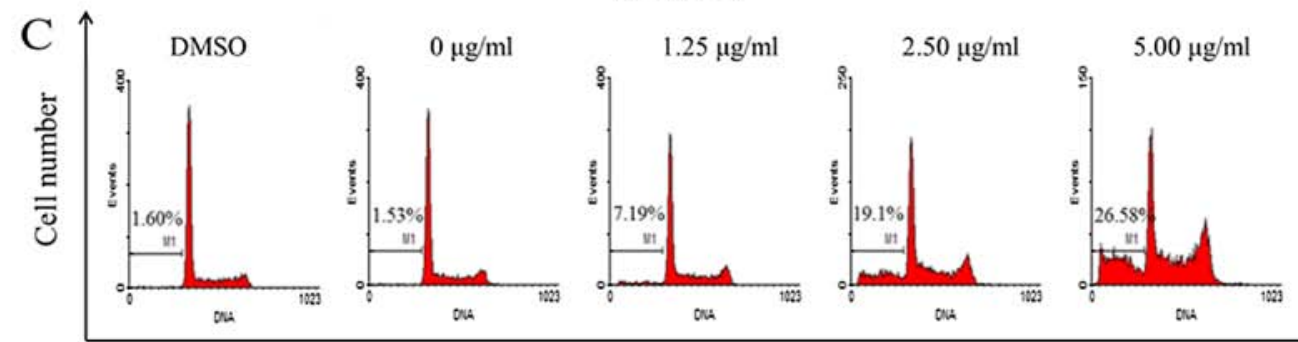

DNA content

D

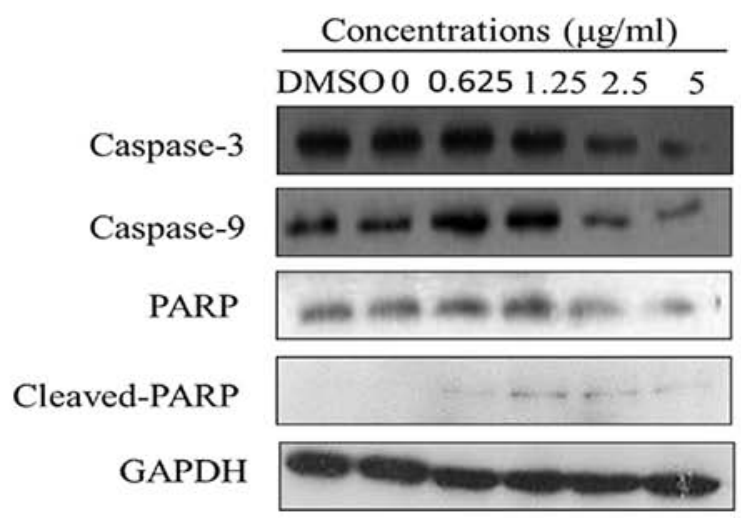

Figure 4. Harmine induces SW620 cell apoptosis in dose-dependent manner. (A) Harmine-induced apoptosis in SW620 cells was assessed by Hoechst 33258 staining. Morphology of the SW620 cells exposed to harmine at different concentrations photographed under a fluorescence microscope (original magnification, x200). (B) SW620 cells were treated with different concentrations of harmine for $48 \mathrm{~h}$, and the staining of cells with Annexin V-FITC and PI was used to distinguish and quantitatively determine the percentage of apoptotic cells. (C) SW620 cells were treated with different concentrations of harmine for $48 \mathrm{~h}$, and PI staining was used to detect the percentage of sub-G1 cells by flow cytometry. (D) The expression levels of apoptosis-associated proteins, caspase- 9 and -3 , and PARP, were analyzed by western blot analysis. The data represent one of three experiments that yielded similar results.

Therefore, we examined whether harmine could inhibit the phosphorylation of downstream targets of Akt. As expected, the phosphorylation levels of FoxO3a and GSK-3 $\beta$ were partially attenuated by harmine in a dose-dependent manner without affecting the amount of total protein in the SW620 cells. Meanwhile, the phosphorylation levels of ERK were also inhibited without affecting the amount of total protein.

\section{Discussion}

In the present study, we demonstrated that harmine dosedependently inhibited the growth of SW620 cells, induced mitochondrial-dependent apoptosis, and arrested the cell cycle in the $\mathrm{S}$ and G2/M phases. More significantly, we demonstrated that inhibition of the ERK and Akt signaling pathways was involved in the antitumor activity of harmine (Fig. 7).

The cell cycle is a common phenomenon of eukaryotic cell division. In terms of the effects on the cell cycle, there are four key checkpoints in cell cycle progression: G1/S, S, G2/M and spindle assembly checkpoints (20). Cyclin-dependent kinase inhibitors (CKIs), p53, Weel and Myt1 play important roles in the regulation of the cell cycle (21). At the G2/M phase checkpoint, Akt downstream proteins, Weel and Myt1 can inhibit the progression of the cell cycle by phosphorylating cyclin- 


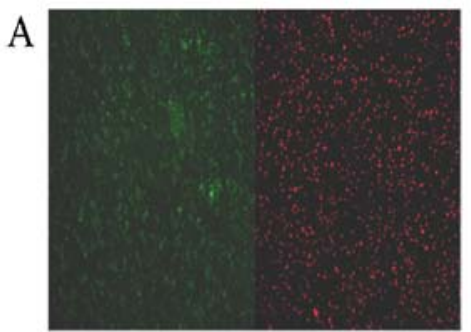

DMSO

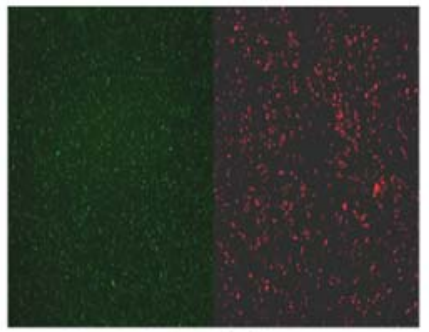

$0 \mu \mathrm{g} / \mathrm{ml}$

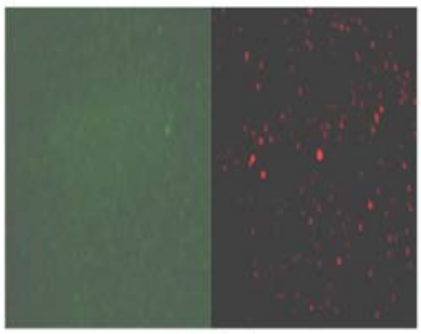

$2.50 \mu \mathrm{g} / \mathrm{ml}$

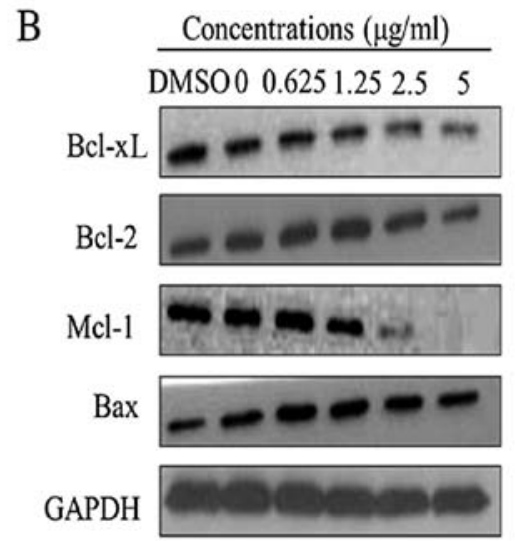

Figure 5. Harmine induces the apoptosis of SW620 cells through the mitochondrial signaling pathway. (A) Change in $\Delta \Psi \mathrm{m}$ was evaluated by fluorescence microscopy. SW620 cells were treated with $2.50 \mu \mathrm{g} / \mathrm{ml}$ harmine for $48 \mathrm{~h}$. (B) Harmine modulated the expression of Bcl-2 family members. SW620 cells were treated with $0,0.625,1.25,2.50$ and $5.00 \mu \mathrm{g} / \mathrm{ml}$ harmine for $48 \mathrm{~h}$, and the expression of Bcl-2 family members was evaluated by western blot analysis. The data were carried out for at least three separate experiments.

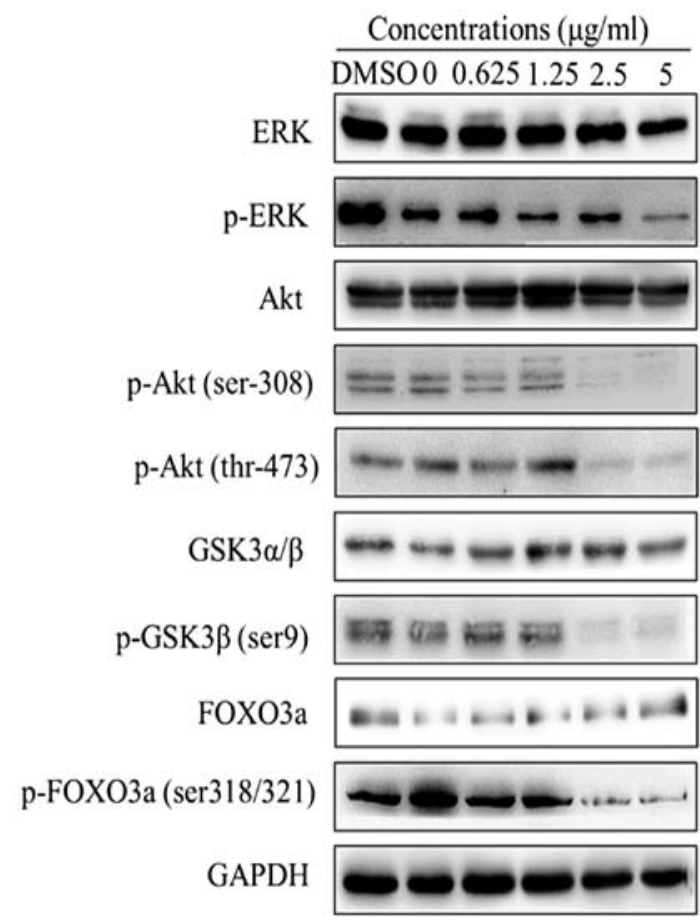

Figure 6. Changes in the Akt and ERK signaling pathway. SW620 cells were treated with different concentrations of harmine for $48 \mathrm{~h}$. Expression levels of the phosphorylation of ERK, Akt and phosphorylation of its downstream targets FoxO3a and GSK-3 $\beta$ were analyzed by western blot analysis. The data represent one of three experiments yielding similar results.

dependent kinases (CDKs) at specific sites, such as the Tyr15 and Thr14 in the ATP binding loop of cdc2 $(22,23)$. In these experiments, the proportions of cells in the $\mathrm{S}$ and $\mathrm{G} 2 / \mathrm{M}$ phases were increased in a dose-dependent manner, as shown by flow cytometric analysis. Western blot analysis showed that harmine downregulated cyclin D1 but upregulated cyclin A, E2 and $\mathrm{B} 1$, in accordance with the flow cytometric analysis results. Harmine changes the cell cycle distribution, and this differs among cell types. Yang et al indicated that harmine treatment resulted in G1 cell cycle arrest in a dose-dependent manner in human breast cancer cells (14), and harmine induced human umbilical vein endothelial cells (HUVECs) to arrest at the $\mathrm{S}$ and G2/M phases (10). Our previous study showed that harmine also blocked the cell cycle at the $\mathrm{S}$ and $\mathrm{G} 2 / \mathrm{M}$ phases in HepG2 cells, similar to our results in SW620 cells (11). Our results also showed that Myt1 and p-cdc2 (Tyr15) were upregulated, and p-Akt was downregulated following treatment with harmine. The upregulation of Myt1 reduced the activity of cdc2 through phosphorylation of Tyr15, which can induce inhibition of the cdc2/cyclin B complex and cell cycle arrest in the S and G2/M phases in SW620 cells.

Four experiments, including Hoechst 33258 staining and fluorescence microscopy; PI staining and flow cytometry; Annexin V-FITC and PI staining and flow cytometry; and western blot analysis for the detection of caspase- 9 and -3 and PARP, revealed that harmine treatment resulted in SW620 cells undergoing apoptosis, and all of the results suggest that the growth inhibition of SW620 cells by harmine is due to its ability to induce apoptosis.

The Bcl-2 family proteins play important roles in the regulation of cellular apoptosis (19). When levels of anti-apoptotic proteins on the mitochondrial membrane, such as $\mathrm{Bcl}-2, \mathrm{Bcl}-\mathrm{xL}$ and Mcl-1, decrease and levels of pro-apoptotic proteins (Bax 


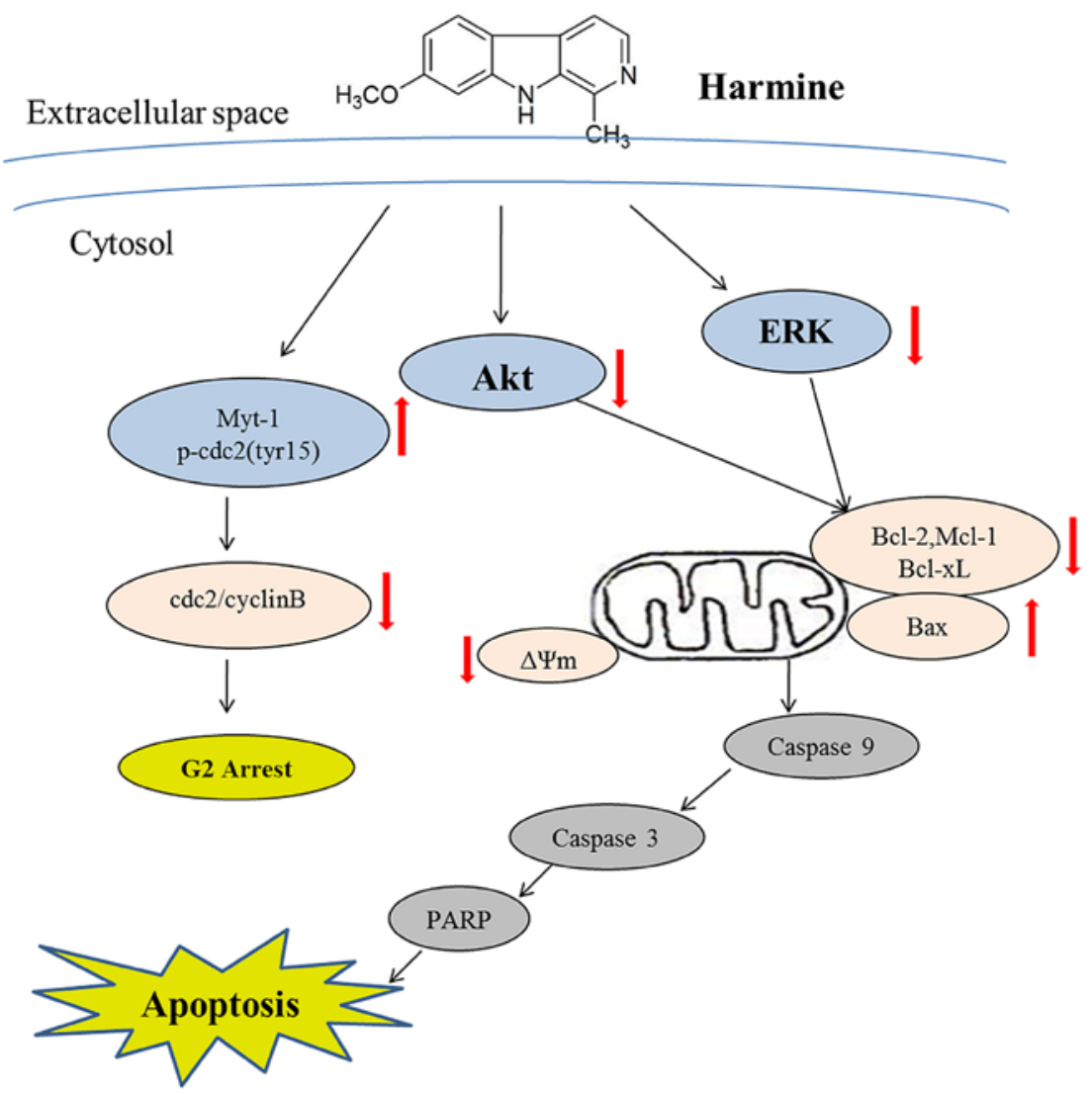

Figure 7. A schematic illustration demonstrating the effects and mechanism of harmine on cell cycle distribution and apoptosis in SW620 cells. Harmine upregulates myt1, phosphorylates cdc2 at Tyr15, and inhibits the activity of the cdc2/cyclin B complex. It also demonstrates that inhibition of ERK and inhibition of PI3K/Akt signaling play important roles in harmine-induced mitochondrial associated apoptosis in SW620 cells.

and Bak) increase or remain unchanged, the ratios of Bcl-2/ $\mathrm{Bax}, \mathrm{Bcl}-\mathrm{xL} / \mathrm{Bax}$ and $\mathrm{Mcl}-1 / \mathrm{Bax}$ decrease, leading to a relative increase in Bax. Bax proteins change their configurations to form oligomers and insert into the mitochondrial membrane as pores, which leads to the release of cytochrome $c$ from the mitochondria and the initiation of the caspase cascade and subsequently to apoptosis $(24,25)$. In our experiments, harmine caused disruption of $\Delta \Psi \mathrm{m}$ and increased the expression of pro-apoptotic Bax but decreased the expression levels of antiapoptotic Bcl-2, Bcl-xL and Mcl-1, leading to the upregulation of the ratios of $\mathrm{Bax} / \mathrm{Bcl}-2$, Bax/Bcl-xL and Bax/Mcl-1. These results demonstrated that harmine induced apoptosis through the mitochondrial pathway in SW620 cells.

The PI3K-Akt signaling pathway is an important intracellular signal transduction pathway. It plays important roles in cell proliferation, cell growth and metabolism, cell survival and angiogenesis by affecting the activity of downstream molecules, and it is closely associated with the development and progression of human tumors. This pathway can directly phosphorylate Bad on Ser136, and this creates a binding site for 14-3-3 proteins, which triggers the release of Bad from its target proteins. Akt can phosphorylate Ser196 on human procaspase-9, and this phosphorylation correlates with a decrease in the protease activity of caspase- 9 in vitro. Akt also phosphorylates MDM2 on Ser166 and Ser186, and this promotes the translocation of MDM2 to the nucleus, where it negatively regulates p53. Other substrates of Akt include FoxO, GSK3, ASK1, TSC2, RAF1, Chk1, IKK $\alpha$, p21 ${ }^{\text {cip1 }}$ and p27 ${ }^{\mathrm{kip} 1}$, which affect cell survival and cell cycle progression (26-28). In the present study, we demonstrated that harmine inhibits Akt kinase activity by decreasing the phosphorylation of Akt on Thr308 and Ser473, and its downstream targets p-FoxO3a and $\mathrm{p}-\mathrm{GSK} 3 \beta$ were markedly decreased in the presence of harmine in vitro.

The targets of FoxO transcription factors include cyclin A, cyclin B, cyclin D, cyclin E, cyclin G2; p15, p19, p21, p27, p130 (cell cycle); and Bim, BNIP3, Fas-ligand, PUMA, PTEN, TRAIL and ATG12 (apoptosis and autophagy). Phosphorylation by Akt inactivated FoxOs, resulting in its accumulation in the cytoplasm and inhibiting its transcriptional function. Through this mechanism, Akt blocks the FoxO-mediated transcription of target genes that promote apoptosis, cell cycle arrest, and metabolic processes (28). Akt also phosphorylates GSK3 isoforms at a highly conserved $\mathrm{N}$-terminal regulatory site (GSK3 $\alpha$-Ser21 and GSK3 $\beta$-Ser9), and this phosphorylation inactivates the kinase. GSK3-mediated phosphorylation of the G1 phase cyclins such as cyclin D and E, and the transcription factors c-jun and c-myc, which all play central roles in the cell cycle transition, targets them for proteasomal degradation, and GSK3 can also target and inhibit Mcl-1 (29,30). In the present study, we found that harmine inhibits the Akt signaling pathway by inhibiting the expression level of p-Akt and its downstream proteins, such as p-FoxO3 and p-GSK3 $\beta$, thus inducing cell cycle arrest and mitochondrial apoptosis.

The activation of ERK1/2 has been shown to inhibit apoptosis in response to a wide range of stimuli. The activation 
of ERK1/2, induced by different initiating signals, results in the phosphorylation of different substrates; thus far, more than 150 substrates have been identified. ERK phosphorylates and inhibits the pro-apoptotic proteins such as caspase- 8 and -9 , Bad, Bim, and STAT3/5, and phosphorylates and activates anti-apoptotic proteins, such as Mcl-1, Bcl-xL, c-Flip, IEX-1, and CBP. Therefore, such as PKB/Akt, activated ERK regulates a number of cellular events, including cell proliferation and survival $(31,32)$. In the present study, we found that harmine downregulated the expression of phosphorylated ERK and induced cell cycle arrest and mitochondrial apoptosis.

In conclusion, the present study demonstrated that harmine induced cell death and growth inhibition in human colorectal carcinoma SW620 cells. Harmine altered the cell cycle distribution by decreasing the proportion of cells in the G0-G1 phase and increasing the proportion in the $\mathrm{S}$ and $\mathrm{G} 2-\mathrm{M}$ phase, partly through upregulation of Myt-1 and p-cdc2 (Tyr15). Harmine induced mitochondrial-related cellular apoptosis by modulating the expression of Bcl-2 family proteins and decreasing mitochondrial transmembrane potential $(\Delta \Psi \mathrm{m})$. We also found that harmine decreased the levels of p-Akt and p-ERK, and this inhibition of the PI3K/Akt and ERK signaling pathways may be involved in harmine-induced cell cycle arrest and apoptosis in SW620 cells.

\section{Acknowledgements}

The present study was supported by grants from the Cultivation Fund of the First Affiliated Hospital of Jinan University (2014203), the Sci-Tech Project Foundation of Guangdong Province in China (2011B031800012), and the Natural Science Foundation of Guangdong Province in China (2014A030313356).

\section{References}

1. Torre LA, Bray F, Siegel RL, Ferlay J, Lortet-Tieulent J and Jemal A: Global cancer statistics, 2012. CA Cancer J Clin 65: 87-108, 2015.

2. Kuebler JP, Wieand HS, O'Connell MJ, Smith RE, Colangelo LH, Yothers G, Petrelli NJ, Findlay MP, Seay TE, Atkins JN, et al: Oxaliplatin combined with weekly bolus fluorouracil and leucovorin as surgical adjuvant chemotherapy for stage II and III colon cancer: Results from NSABP C-07. J Clin Oncol 25: 2198-2204, 2007.

3. Patel K, Gadewar M, Tripathi R, Prasad SK and Patel DK: A review on medicinal importance, pharmacological activity and bioanalytical aspects of beta-carboline alkaloid 'Harmine'. Asian Pac J Trop Biomed 2: 660-664, 2012.

4. Hamsa TP and Kuttan G: Harmine inhibits tumour specific neo-vessel formation by regulating VEGF, MMP, TIMP and pro-inflammatory mediators both in vivo and in vitro. Eur J Pharmacol 649: 64-73, 2010.

5. Zhao L and Wink M: The $\beta$-carboline alkaloid harmine inhibits telomerase activity of MCF-7 cells by down-regulating hTERT mRNA expression accompanied by an accelerated senescent phenotype. PeerJ 1: e174, 2013.

6. Hamsa TP and Kuttan G: Harmine activates intrinsic and extrinsic pathways of apoptosis in B16F-10 melanoma. Chin Med 6: 11,2011.

7. Lala S, Pramanick S, Mukhopadhyay S, Bandyopadhyay S and Basu MK: Harmine: Evaluation of its antileishmanial properties in various vesicular delivery systems. J Drug Target 12: 165-175, 2004.

8. Hudson JB, Graham EA and Towers GH: Antiviral effect of harmine, a photoactive beta-carboline alkaloid. Photochem Photobiol 43: 21-26, 1986.
9. Göckler N, Jofre G, Papadopoulos C, Soppa U, Tejedor FJ and Becker W: Harmine specifically inhibits protein kinase DYRK1A and interferes with neurite formation. FEBS J 276: 6324-6337, 2009.

10. Dai F, Chen Y, Song Y, Huang L, Zhai D, Dong Y, Lai L, Zhang T, Li D, Pang X, et al: A natural small molecule harmine inhibits angiogenesis and suppresses tumour growth through activation of p53 in endothelial cells. PLoS One 7: e52162, 2012.

11. Cao MR, Li Q, Liu ZL, Liu HH, Wang W, Liao XL, Pan YL and Jiang JW: Harmine induces apoptosis in HepG2 cells via mitochondrial signaling pathway. Hepatobiliary Pancreat Dis Int 10: 599-604, 2011.

12. Boeira JM, Viana AF, Picada JN and Henriques JA: Genotoxic and recombinogenic activities of the two beta-carboline alkaloids harman and harmine in Saccharomyces cerevisiae. Mutat Res 500: 39-48, 2002.

13. Zhang H, Sun K, Ding J, Xu H, Zhu L, Zhang K, Li X and Sun W: Harmine induces apoptosis and inhibits tumor cell proliferation, migration and invasion through down-regulation of cyclooxygenase-2 expression in gastric cancer. Phytomedicine 21: 348-355, 2014.

14. Yang X, Wang W, Qin JJ, Wang MH, Sharma H, Buolamwini JK, Wang $\mathrm{H}$ and Zhang R: JKA97, a novel benzylidene analog of harmine, exerts anti-cancer effects by inducing G1 arrest, apoptosis, and p53-independent up-regulation of p21. PLoS One 7: e34303, 2012.

15. Song Y, Kesuma D, Wang J, Deng Y, Duan J, Wang JH and Qi RZ: Specific inhibition of cyclin-dependent kinases and cell proliferation by harmine. Biochem Biophys Res Commun 317: 128-132, 2004.

16. Wang W, Liao XL, Chen JH, Li DD, Lin CL, Yan YX, Tang YH and Jiang JW: Sodium valproate induces mitochondria-dependent apoptosis in human hepatoblastoma cells. Chin Med J 124: 2167-2172, 2011.

17. Tan ML, Ooi JP, Ismail N, Moad AI and Muhammad TS: Programmed cell death pathways and current antitumor targets. Pharm Res 26: 1547-1560, 2009.

18. Qin S, Yang C, Li S, Xu C, Zhao Y and Ren H: Smac: Its role in apoptosis induction and use in lung cancer diagnosis and treatment. Cancer Lett 318: 9-13, 2012.

19. Zhou F, Yang Y and Xing D: Bcl-2 and Bcl-xL play important roles in the crosstalk between autophagy and apoptosis. FEBS J 278: 403-413, 2011.

20. Sánchez I and Dynlacht BD: New insights into cyclins, CDKs, and cell cycle control. Semin Cell Dev Biol 16: 311-321, 2005.

21. Besson A, Dowdy SF and Roberts JM: CDK inhibitors: Cell cycle regulators and beyond. Dev Cell 14: 159-169, 2008.

22. Berry LD and Gould KL: Regulation of Cdc2 activity by phosphorylation at T14/Y15. Prog Cell Cycle Res 2: 99-105, 1996.

23. Fattaey A and Booher RN: Myt1: A Wee1-type kinase that phosphorylates Cdc2 on residue Thr14. Prog Cell Cycle Res 3: 233-240, 1997.

24. Tait SW and Green DR: Mitochondria and cell death: Outer membrane permeabilization and beyond. Nat Rev Mol Cell Biol 11: 621-632, 2010.

25. Adams JM and Cory S: The Bcl-2 apoptotic switch in cancer development and therapy. Oncogene 26: 1324-1337, 2007.

26. Manning BD and Cantley LC: AKT/PKB signaling: Navigating downstream. Cell 129: 1261-1274, 2007.

27. Faes S and Dormond O: PI3K and AKT: Unfaithful partners in cancer. Int J Mol Sci 16: 21138-21152, 2015.

28. Zhang X, Tang N, Hadden TJ and Rishi AK: Akt, FoxO and regulation of apoptosis. Biochim Biophys Acta 1813: 1978-1986, 2011.

29. Luo J: Glycogen synthase kinase 3beta (GSK3beta) in tumorigenesis and cancer chemotherapy. Cancer Lett 273: 194-200, 2009.

30. Phukan S, Babu VS, Kannoji A, Hariharan R and Balaji VN: GSK3beta: Role in therapeutic landscape and development of modulators. Br J Pharmacol 160: 1-19, 2010.

31. Samatar AA and Poulikakos PI: Targeting RAS-ERK signalling in cancer: Promises and challenges. Nat Rev Drug Discov 13: 928-942, 2014.

32. Peng S, Zhang Y, Zhang J, Wang H and Ren B: ERK in learning and memory: A review of recent research. Int J Mol Sci 11: 222-232, 2010. 